This is an electronic reprint of the original article. This reprint may differ from the original in pagination and typographic detail.

Author(s): Tarvainen, Olli; Laulainen, Janne; Komppula, Jani; Kronholm, Risto; Kalvas, Taneli; Koivisto, Hannu; Izotov, I.; Mansfeld, D.; Skalyga, V.

Title: $\quad$ Limitations of electron cyclotron resonance ion source performances set by kinetic plasma instabilities

Year: $\quad 2015$

Version:

Please cite the original version:

Tarvainen, O., Laulainen, J., Komppula, J., Kronholm, R., Kalvas, T., Koivisto, H., Izotov, I., Mansfeld, D., \& Skalyga, V. (2015). Limitations of electron cyclotron resonance ion source performances set by kinetic plasma instabilities. Review of Scientific Instruments, 86(2), Article 023301. https://doi.org/10.1063/1.4906804

All material supplied via JYX is protected by copyright and other intellectual property rights, and duplication or sale of all or part of any of the repository collections is not permitted, except that material may be duplicated by you for your research use or educational purposes in electronic or print form. You must obtain permission for any other use. Electronic or print copies may not be offered, whether for sale or otherwise to anyone who is not an authorised user. 


\section{AIP $\mid \begin{aligned} & \text { Review of } \\ & \text { Scientific Instruments }\end{aligned}$}

\section{Limitations of electron cyclotron resonance ion source performances set by kinetic plasma instabilities}

O. Tarvainen, J. Laulainen, J. Komppula, R. Kronholm, T. Kalvas, H. Koivisto, I. Izotov, D. Mansfeld, and V. Skalyga

Citation: Review of Scientific Instruments 86, 023301 (2015); doi: 10.1063/1.4906804

View online: http://dx.doi.org/10.1063/1.4906804

View Table of Contents: http://scitation.aip.org/content/aip/journal/rsi/86/2?ver=pdfcov

Published by the AIP Publishing

\section{Articles you may be interested in}

Suppression of cyclotron instability in Electron Cyclotron Resonance ion sources by two-frequency heating Phys. Plasmas 22, 083509 (2015); 10.1063/1.4928428

Influence of microwave driver coupling design on plasma density at Testbench for lon sources Plasma Studies, a $2.45 \mathrm{GHz}$ Electron Cyclotron Resonance Plasma Reactor

Rev. Sci. Instrum. 85, 033310 (2014); 10.1063/1.4869343

Electron cyclotron resonance charge breeder ion source simulation by MCBC and GEMa)

Rev. Sci. Instrum. 79, 02B906 (2008); 10.1063/1.2816961

Dynamic simulations of the interchange instability, ion production, and electron heating processes in an electron cyclotron resonance ion source plasma

Rev. Sci. Instrum. 71, 846 (2000); 10.1063/1.1150309

Limitations to the plasma energy and density in electron cyclotron resonance ion sources

Phys. Plasmas 6, 3408 (1999); 10.1063/1.873581

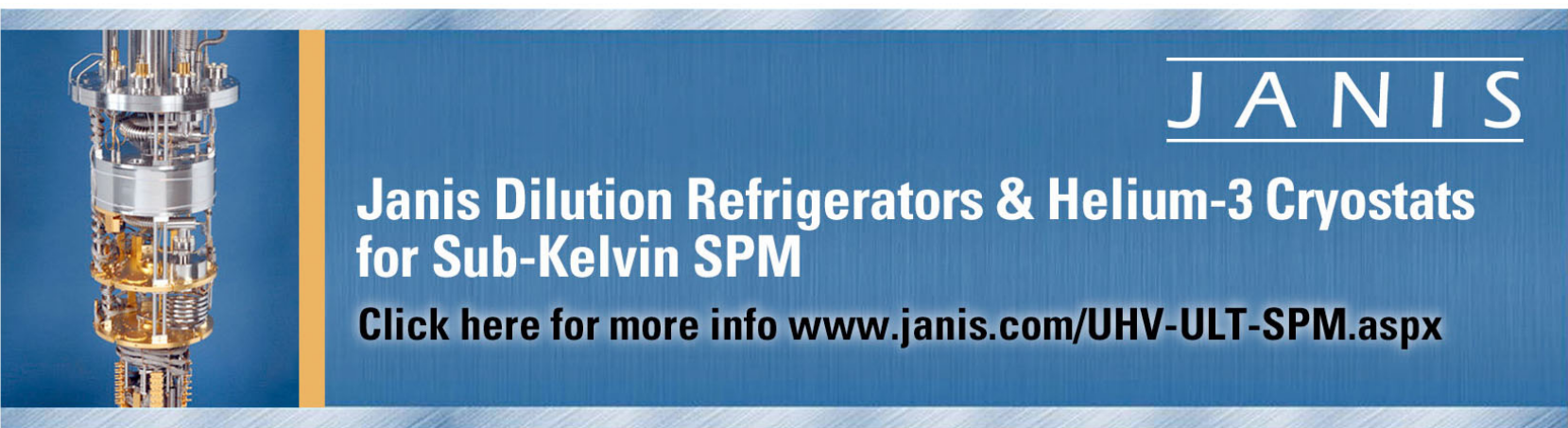




\title{
Limitations of electron cyclotron resonance ion source performances set by kinetic plasma instabilities
}

\author{
O. Tarvainen, ${ }^{1, a)}$ J. Laulainen, ${ }^{1}$ J. Komppula, ${ }^{1}$ R. Kronholm, ${ }^{1}$ T. Kalvas, ${ }^{1}$ H. Koivisto, ${ }^{1}$ \\ I. Izotov, ${ }^{2}$ D. Mansfeld, ${ }^{2}$ and V. Skalyga ${ }^{2,3}$ \\ ${ }^{1}$ Department of Physics, University of Jyväskylä, 40500 Jyväskylä, Finland \\ ${ }^{2}$ Institute of Applied Physics, RAS, 46 Ul'yanova St., 603950 Nizhny Novgorod, Russian Federation \\ ${ }^{3}$ Lobachevsky State University of Nizhny Novgorod (UNN), 23 Gagarina St., \\ 603950 Nizhny Novgorod, Russian Federation
}

(Received 4 December 2014; accepted 15 January 2015; published online 4 February 2015)

\begin{abstract}
Electron cyclotron resonance ion source (ECRIS) plasmas are prone to kinetic instabilities due to anisotropy of the electron energy distribution function stemming from the resonant nature of the electron heating process. Electron cyclotron plasma instabilities are related to non-linear interaction between plasma waves and energetic electrons resulting to strong microwave emission and a burst of energetic electrons escaping the plasma, and explain the periodic oscillations of the extracted beam currents observed in several laboratories. It is demonstrated with a minimum- $B 14 \mathrm{GHz}$ ECRIS operating on helium, oxygen, and argon plasmas that kinetic instabilities restrict the parameter space available for the optimization of high charge state ion currents. The most critical parameter in terms of plasma stability is the strength of the solenoid magnetic field. It is demonstrated that due to the instabilities the optimum $B_{\mathrm{min}}$-field in single frequency heating mode is often $\leq 0.8 B_{\mathrm{ECR}}$, which is the value suggested by the semiempirical scaling laws guiding the design of modern ECRISs. It is argued that the effect can be attributed not only to the absolute magnitude of the magnetic field but also to the variation of the average magnetic field gradient on the resonance surface. (C) 2015 AIP Publishing LLC. [http://dx.doi.org/10.1063/1.4906804]
\end{abstract}

\section{INTRODUCTION}

Electron Cyclotron Resonance Ion Sources (ECRIS) are used in large-scale accelerator facilities for the production of highly charged heavy ion beams of stable and radioactive elements. ${ }^{1,2}$ The magnetic field of an ECRIS is a superposition of solenoid and sextupole fields forming so-called minimum-B topology. The magnetic field configuration provides a closed surface for resonant energy transfer from microwaves to the plasma electrons, enables sufficient plasma confinement, and suppresses magnetohydrodynamic instabilities. ${ }^{3}$

The effect of the field structure on the performance of ECR ion sources has been studied extensively. The results have been summarized in the form of semiempirical scaling laws expressed as ${ }^{4,5} \frac{B_{\text {inj }}}{B_{\mathrm{ECR}}} \geq 4, \frac{B_{\mathrm{ext}}}{B_{\mathrm{ECR}}} \geq 2, \frac{B_{\mathrm{rad}}}{B_{\mathrm{ECR}}} \geq 2$, and $\frac{B_{\mathrm{min}}}{B_{\mathrm{ECR}}}$ $\approx 0.8$, where $B_{\text {inj }}$ and $B_{\text {ext }}$ are the field maxima at injection and extraction, $B_{\text {rad }}$ is the maximum radial field on the magnetic pole, $B_{\min }$ the minimum field value in the plasma chamber, and $B_{\mathrm{ECR}}$ the resonance field. Modern high-performance ECR ion sources are typically designed based on the given set of semiempirical equations. The scaling laws do not take into account the effect of the magnetic field gradient on the resonance surface. It has been shown that the field gradient plays a pivotal role in determining the electron energy gain in single resonance crossing and hence the development of the electron energy distribution. ${ }^{6-8}$

ECRIS plasmas are strongly anisotropic ${ }^{9,10}$ with the maximum electron energy reaching values up to $1 \mathrm{MeV} .{ }^{11}$

a) olli.tarvainen@jyu.fi
Such non-equilibrium plasmas are prone to kinetic instabilities driven by hot electrons whose transverse velocity $\vec{v} \perp \vec{B}$ dominates over the longitudinal one $\vec{v} \| \vec{B} \cdot{ }^{12,13}$ The instabilities lead to ms-scale oscillation of the extracted beam current with a maximum amplitude of several tens of percents. ${ }^{14}$ In this paper, we focus on demonstrating that electron cyclotron instabilities restrict the tuning range of ECR ion source magnetic field and hence limit the extracted currents of highly charged ions. In particular, it is demonstrated that due to the instabilities, the optimum $B_{\min }$-field is often $<0.8 B_{\mathrm{ECR}}$ suggested by the scaling laws.

\section{THEORETICAL BACKGROUND}

We have, recently, reported ${ }^{14}$ an experimental observation of electron cyclotron instabilities in a minimum-B ECRIS plasma sustained by $14 \mathrm{GHz}$ microwave radiation in cw-mode. A characteristic feature of electron cyclotron plasma instabilities is the emission of microwaves. ${ }^{12}$ The volumetric energy of the microwave emission $E_{\mu}$ can be described by the modedependent growth and damping rates $\gamma$ and $\delta$ as

$$
\frac{d E_{\mu}}{d t} \approx\langle\gamma-\delta\rangle E_{\mu},
$$

i.e., the intensity of the microwave emission is an exponential function of the difference of the growth and damping rates. The instabilities are triggered by the anisotropy of the electron velocity distribution (EVD), and their volumetric growth rate is proportional to the magnetic field through the local electron cyclotron frequency and the ratio of hot and cold 

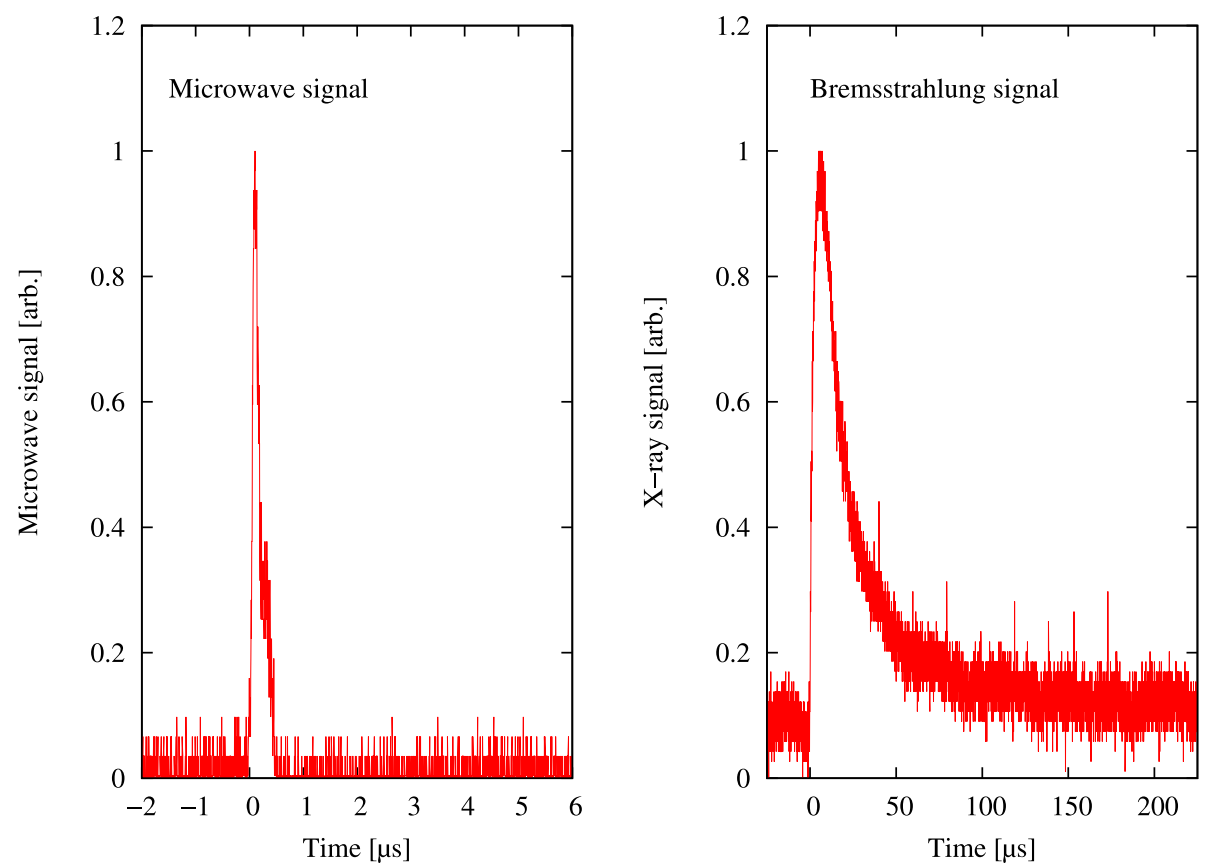

FIG. 1. Examples of microwave and bremsstrahlung signals associated with an onset of the periodic plasma instability.

electron densities, i.e., $\gamma \propto \omega_{c e} \frac{N_{e} \text {,hot }}{N_{e, \text { cold }}},{ }^{15,16}$ where $\omega_{c e}=\frac{e B}{m_{e}}$. The damping rate is determined by volumetric absorption of the wave energy by the collisional background plasma and external (wall) losses, i.e., $\delta \propto v_{e}+R$ where $R$ represents the reflection/wall loss term (for further discussion see Ref. 17). In unstable operating conditions, i.e., when $\gamma>\delta$, hot electrons interacting with the amplifying plasma wave emit microwave radiation and are expelled into the loss cone. The increased flux of electrons from the trap results in a burst of wall bremsstrahlung ${ }^{14}$ and stabilization of the plasma due to reduced density of hot electrons. However, the instability appears again at an interval corresponding to the characteristic time required to create necessary anisotropy of the EVD and leads to periodic oscillations of the extracted ion currents. ${ }^{14,18}$ Representative examples of diagnostics signals, i.e., microwave and bremsstrahlung emission, and beam current oscillations associated

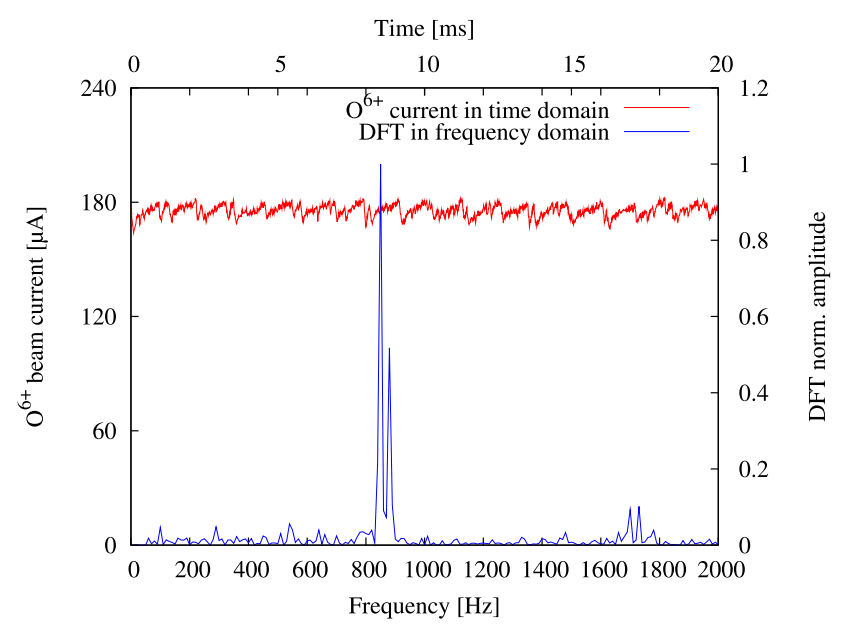

FIG. 2. An example of $\mathrm{O}^{6+}$ beam current signal in unstable operation regime of an ECRIS and corresponding Discrete Fourier Transform (DFT) spectrum showing the characteristic period of the oscillation. Each drop of the beam current corresponds to an onset of electron cyclotron instability. with an onset of the electron cyclotron instability are shown for illustration purposes in Figs. 1 and 2. The data have been obtained with the JYFL $14 \mathrm{GHz}$ ECRIS. ${ }^{19}$

From the practical point-of-view, it is useful to relate (qualitatively) the tuning parameters of an ECRIS, i.e., Bfield configuration, microwave power, and neutral gas pressure, with the growth and damping rates of the electron cyclotron instabilities. It has been shown theoretically ${ }^{6}$ and through bremsstrahlung diagnostics ${ }^{21,22}$ that the maximum energy of the plasma electrons increases with decreasing magnetic field gradient at the resonance and with increasing microwave power indicating higher growth rate of the instabilities. The most critical parameter affecting the occurrence of the cyclotron instabilities is the (solenoid) magnetic field configuration. ${ }^{14}$ It can be also hypothesized that increasing the neutral gas pressure results to higher (inelastic) collision frequency and subsequent enhancement of the instability damping rate as reported and discussed in Refs. 14 and 23. Furthermore, it has been observed ${ }^{23}$ that the instability threshold shifts towards higher $B_{\min } / B_{\mathrm{ECR}}$-ratio with increasing atomic number of the plasma species, which is probably due to inelastic collisions enhancing the damping rate of the instabilities.

\section{EXPERIMENTAL SETUP}

The experimental data discussed in this article were taken with the room-temperature A-ECR type JYFL $14 \mathrm{GHz}$ ECRIS $^{19}$ operated with a single frequency $(14.085 \mathrm{GHz})$ in continuous $(\mathrm{CW})$ mode. The magnetic field of the ion source is generated by two solenoid coils and a permanent magnet sextupole and it fulfills the semi-empirical scaling laws discussed above. The magnetic field strength can be adjusted by varying the solenoid coil currents, which affects the injection and extraction mirror ratios as well as the Bminimum. The strength of the sextupole field on the plasma 
chamber wall at the magnetic pole is $1.07 \mathrm{~T} .{ }^{20}$ It is of note that adjusting the solenoid coil currents affects also the radial mirror ratio due to the superposition of the sextupole field and the radial component of the solenoid field, i.e., the mirror ratios cannot be tuned independently. The diagnostics setup applied for detecting electron cyclotron instabilities has been thoroughly described in the literature. ${ }^{14}$ Since the focus of the experiments described in this paper is on demonstrating that the instabilities limit the tuning range of ion source parameters and hence optimization of the extracted currents of highly charged ions, the experimental setup was simplified to include only a Bismuth germanate (BGO) scintillator coupled with a Na-doped CsI (300-600 nm) current-mode photomultiplier tube (PMT) at a distance of $120 \mathrm{~mm}$ from the radial port of the ECRIS, and a Faraday cup located near the downstream focal point of an $m / q$-analyzing magnet. The scintillator was used for detecting periodic bursts of bremsstrahlung indicating the existence of cyclotron instabilities (see Fig. 1 in Sec. II) and, thus, distinguishing between stable and unstable operation regimes. The Faraday cup was used for measuring time-averaged beam currents of different charge states of helium, oxygen, and argon ion beams. The data were taken by
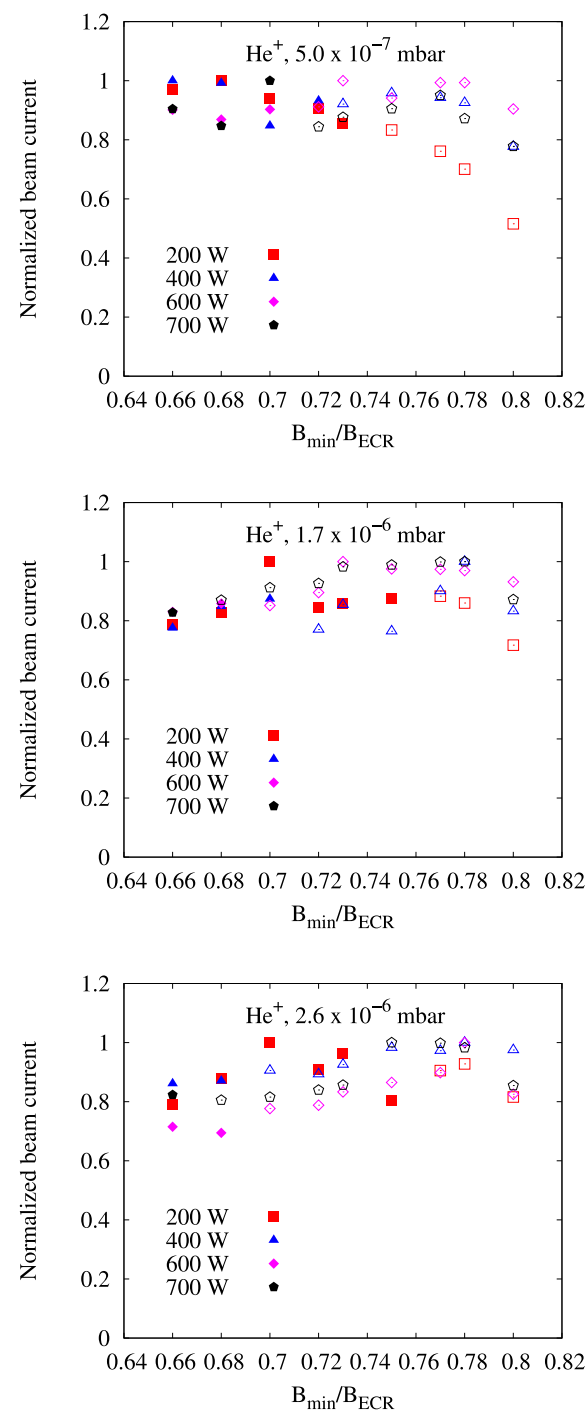

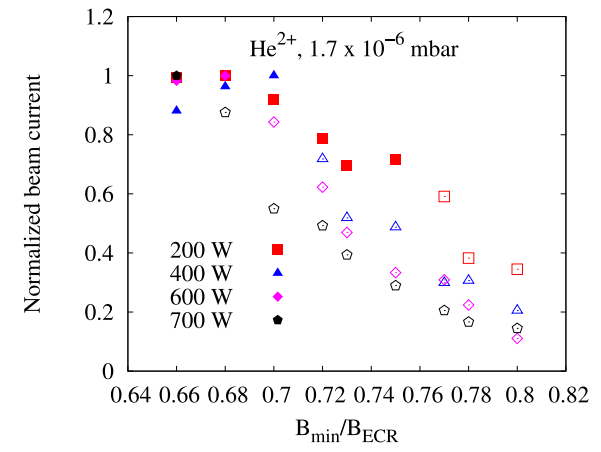

sweeping the solenoid currents (equal current in both coils) across the transition from stable to unstable operation regime at various microwave powers and neutral gas pressures with $10 \mathrm{kV}$ source potential. The voltages of the focusing einzel lenses of the extraction system ${ }^{24}$ were kept constant during the magnetic field sweep at each power/pressure combination while the beamline optics, i.e., two solenoids and the analyzing magnet, were optimized for all data points. The voltage of the biased disc at the injection was set to a constant value of $-150 \mathrm{~V}$.

\section{EXPERIMENTAL RESULTS}

It is considered beneficial for high charge state ion production to improve the plasma confinement and enhance the electron density and average energy by increasing the magnetic field strength and/or microwave power. Such conditions, however, make the plasma prone to instabilities that can limit the average extracted currents of highly charged ions. This is demonstrated in Figs. 3, 4, and 5 showing the normalized temporally averaged currents (over several seconds), of certain
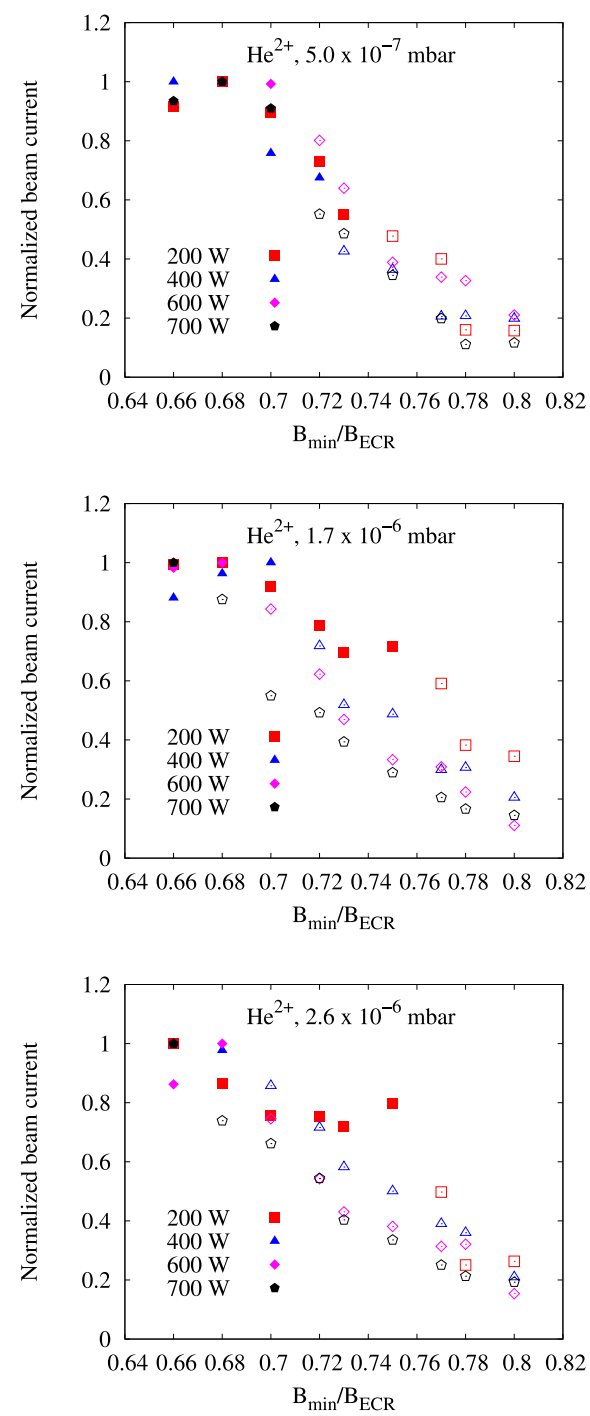

FIG. 3. Normalized currents of $\mathrm{He}^{+}$and $\mathrm{He}^{2+}$ ion beams as a function of the $\boldsymbol{B}_{\mathrm{min}} / \boldsymbol{B}_{\mathrm{ECR}}$-ratio. Solid symbols correspond to stable and open symbols to unstable operation regime. 

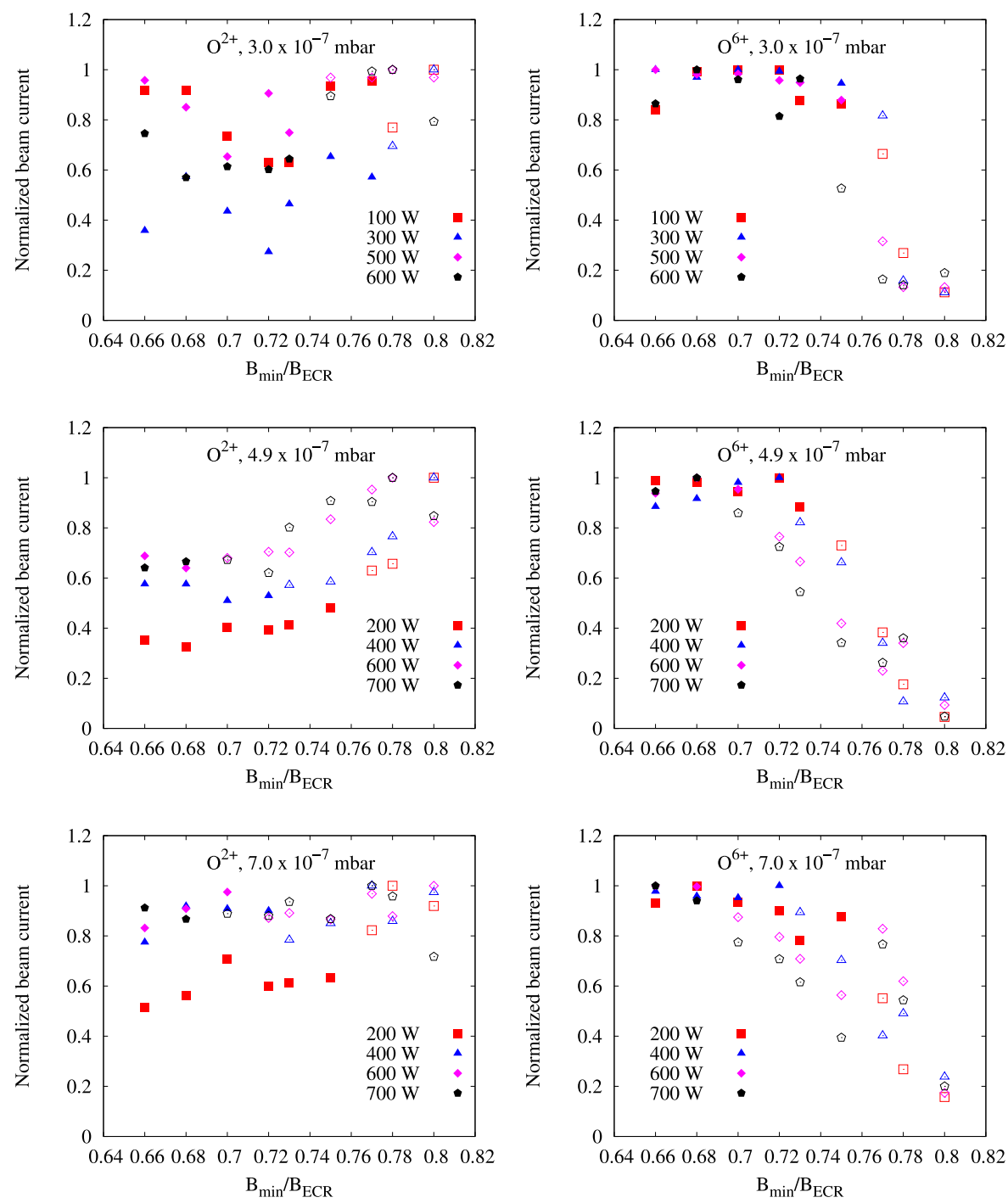

FIG. 4. Normalized currents of $\mathrm{O}^{2+}$ and $\mathrm{O}^{6+}$ ion beams as a function of the $B_{\min } / B_{\mathrm{ECR}}$-ratio. Solid symbols correspond to stable and open symbols to unstable operation regime.

charge states of helium, oxygen, and argon as a function of the $B_{\min } / B_{\mathrm{ECR}}$-ratio at different microwave powers and neutral gas pressures that are typical for the JYFL $14 \mathrm{GHz}$ ECRIS operation. The data sets chosen for display represent only a small fraction of the data (collected at various microwave powers and neutral gas pressures for several charge states of the given elements) that supports the conclusions made hereafter. The normalization is carried out independently for each data set, i.e., magnetic field sweep, measured at given incident microwave power. The normalization is carried out in order to eliminate the effect of microwave power, namely, the increase of extracted currents of highly charged ions with increasing power, from the presented data. The purpose of the normalization is to highlight the fact that the occurrence of electron cyclotron instabilities restricts the parameter space available for the optimization of high charge state ion currents due to the existence of instability threshold, which depends on the magnetic field strength. The indicated neutral particle pressures are gas calibrated readings of an ionization gauge located outside the plasma chamber and connected to it through a radial diagnostics port. The given pressure values are measured without igniting the plasma. The reflected power (measured at the klystron amplifier) was less than 5\% of the incident power for all data points shown in this paper.

The data obtained with helium show that the extracted currents of $\mathrm{He}^{2+}$ (the highest possible charge state) decrease drastically with increasing $B_{\min } / B_{\mathrm{ECR}}$-ratio above the instability threshold. Similar effect can be observed for oxygen charge states $\geq \mathrm{O}^{4+}$ with $\mathrm{O}^{6+}$ serving as an illustrative example. On the contrary, the extracted currents of low charge states, such as $\mathrm{O}^{2+}$ shown here, are higher in the unstable operation regime indicating a substantial shift of the ion charge state distribution in the plasma. In the case of argon, charge states $\geq \mathrm{Ar}^{8+}$ suffer from the instabilities as demonstrated using $\mathrm{Ar}^{9+}$ and $\mathrm{Ar}^{12+}$ as examples. Several data series (pressure-power combinations) show that the extracted currents of highly charged ions tend to increase with increasing $B_{\min } / B_{\mathrm{ECR}}$-ratio until the instability threshold is reached. In the unstable regime, it is impossible to restore the source performance by varying the injected power or neutral gas pressure. Thus, the data indicate that electron cyclotron instabilities are limiting ECRIS performances. Moreover, it can be observed that in single frequency 

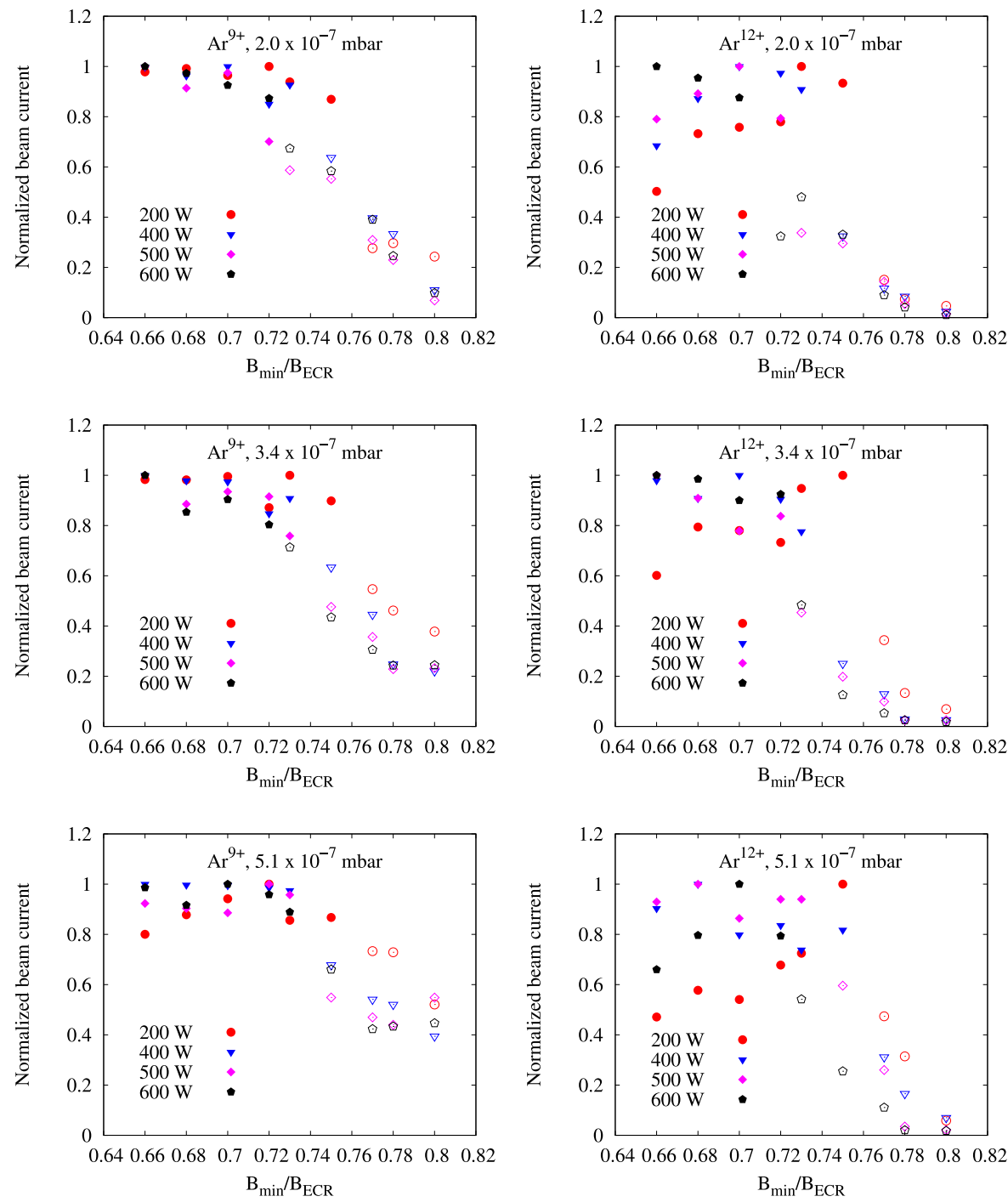

FIG. 5. Normalized currents of $\mathrm{Ar}^{9+}$ and $\mathrm{Ar}^{12+}$ ion beams as a function of the $B_{\min } / B_{\mathrm{ECR}}$-ratio. Solid symbols correspond to stable and open symbols to unstable operation regime.

operation, the instability threshold is systematically reached at values $0.70 \leq \frac{B_{\min }}{B_{\mathrm{ECR}}} \leq 0.75$ which is below the optimum $B_{\mathrm{min}} / B_{\mathrm{ECR}}$-ratio of 0.8 suggested by the ECRIS scaling laws. It is of note that the effect of the instabilities on the extracted beam currents is pronounced for the highest charge states.

It has been, previously, reported by Arai et al. ${ }^{25}$ Leitner et al. ${ }^{26}$ and Nakagawa et al. ${ }^{27}$ that the ECRIS performances peak at $0.70<\frac{B_{\min }}{B_{\mathrm{ECR}}}<0.80$. Furthermore, Hitz et al. ${ }^{5}$ show that the optimum $B_{\text {ext }} / B_{\text {rad }}$-ratio is $0.80-0.95$. The corresponding ratio for the JYFL $14 \mathrm{GHz}$ ECRIS at the instability threshold is 0.9 (using the sextupole field strength as $B_{\mathrm{rad}}$ ). Thus, the present results highlighting the effect of kinetic instabilities on ECRIS performances are in good agreement with earlier studies focusing on measuring the beam currents only.

\section{DISCUSSION}

The charge state dependence of the effect of the instabilities can be explained by the fact that periodic disturbances of the plasma confinement occur at temporal intervals, which are shorter than the confinement time (or production time) of the high charge state ions in stable operating regime. Douysset et al. have measured the argon ion confinement times in quiescent ECRIS plasmas. ${ }^{10}$ The reported data indicate that the argon ion confinement times range from $0.1 \mathrm{~ms}$ $\left(\mathrm{Ar}^{1+}\right)$ to $3.0 \mathrm{~ms}\left(\mathrm{Ar}^{16+}\right)$ depending almost linearly on the charge state. Based on theoretical estimates, ${ }^{10,28}$ it can be expected that the confinement times of other plasma species are on the same order of magnitude. The repetition rate of the electron cyclotron instabilities varies typically from $400 \mathrm{~Hz}$ to $2.5 \mathrm{kHz},{ }^{14,29}$ which corresponds to temporal period of 0.4-2.5 ms. It is, therefore, expected that the extracted currents of high charge state ions, requiring longer confinement and even longer ionization/(density) saturation times, ${ }^{30}$ suffer from the periodic disturbances as indicated by the present systematic data. On the other hand, periodic losses of energetic electrons decrease the ionization rate of highly charged ions. It has been observed ${ }^{14}$ that at magnetic fields exceeding the threshold value, the repetition rate of the instabilities increases with increasing magnetic field strength and microwave power. In other words, it is expected that the extracted currents of highly charged ions decrease monotonically with increasing 
magnetic field in the unstable operation regime. Such behavior is clearly observed in Figs. 3, 4, and 5. The effect of the instabilities on high charge state ion production can also be viewed from the point-of-view of energy lifetime in ECRIS plasmas as discussed by Geller ${ }^{1}$ (pp. 273-275). It has been concluded that if the energy lifetime is smaller than $10^{-3} \mathrm{~s}$, the necessary electron power flux for high charge state ionization cannot be achieved with a microwave power on the order of $\mathrm{kW}$, which is technically feasible for ECRISs. The observed instabilities limit the energy lifetime by inducing hot electron losses at a rate that exceeds the "nominal" electron loss rate due to collisional velocity space diffusion and/or RF pitch angle scattering ${ }^{31,32}$ in quiescent plasmas.

Let us now turn the attention to the root cause of the instabilities, i.e., the anisotropy of the electron velocity distribution function (EVDF). The thread of the following arguments is that the energy gain of the electrons in a single resonance crossing and the plasma confinement is dictated by the magnetic field topology of the ECRIS depicted schematically in Fig. 6.

So far, we have adopted the commonly used practice to quantify the strength of the ECRIS B-field by the ratio of the minimum field to the resonance field $B_{\min } / B_{\mathrm{ECR}}$, where $B_{\mathrm{ECR}}$ for cold electrons is $0.5 \mathrm{~T}$ for $14 \mathrm{GHz}$ microwaves. However, the given ratio is not directly linked with the electron energy gain. It can be shown ${ }^{6}$ that the volumetric power absorption $\left\langle P_{\text {abs }}\right\rangle$ in absence of collisions is proportional to the difference between the resonance field and the local magnetic field as $\left\langle P_{\mathrm{abs}}\right\rangle \propto\left(B_{\mathrm{ECR}}-B\right)^{-2}$. Therefore, the electron energy gain depends strongly on the magnetic field gradient at the resonance, $\nabla B_{\mathrm{ECR}}$ as discussed in numerous papers. ${ }^{6-8}$ The problematic aspect of interpreting experimental data in terms of $\nabla B_{\mathrm{ECR}}$ is that the gradient is not constant over the closed ECR surface. Furthermore, the effective width of the resonance depends only on the component of the magnetic field gradient, which is parallel to the magnetic field. ${ }^{6}$ In order to circumvent these problems of characterizing the magnetic field topology, it is customary to report the on-axis gradient values of the solenoid field. Although the on-axis gradients are a measure of the solenoid field, they should not be considered to correlate directly with the electron energy

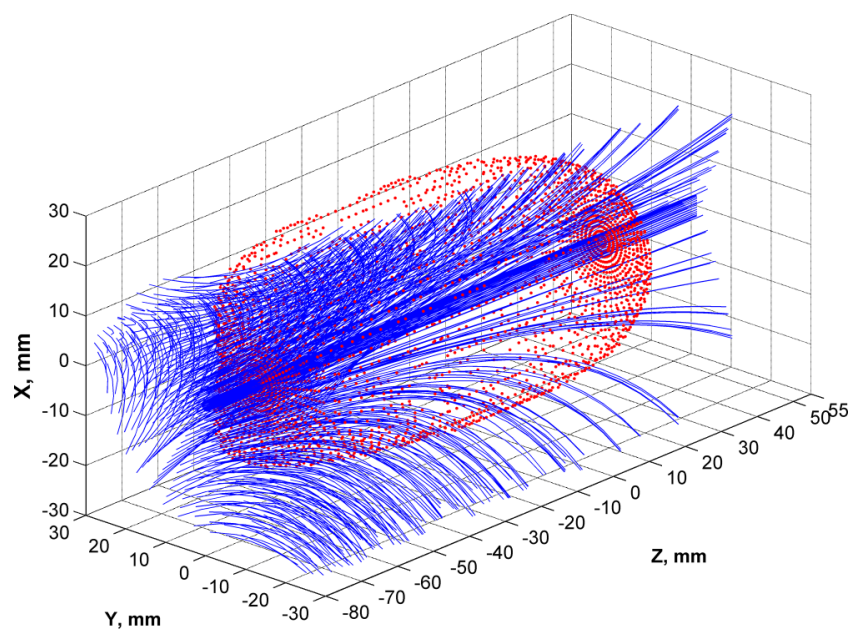

FIG. 6. A schematic presentation of the JYFL 14 GHz ECRIS magnetic field. The magnetic field lines are depicted with solid lines and the ECR-surface for cold electrons with dots. distribution. A preferred approach is to plot the distribution of the parallel magnetic field gradient component, i.e., $\frac{\vec{B}}{|\vec{B}|} \cdot \nabla \vec{B}$ and/or calculate the average parallel gradient $\left\langle\nabla B_{\mathrm{ECR}}\right\rangle_{\|}$across the whole ECR-surface. Furthermore, it must be kept in mind that due to relativistic effects the resonance field value and the corresponding magnetic field gradient depend on the electron energy. Figure 7 shows the distribution of parallel resonance magnetic field gradient components of the JYFL $14 \mathrm{GHz}$ ECRIS for three different electron energies and magnetic field configurations. The magnetic field configurations chosen for display correspond to stable operation regime with $\frac{B_{\min }}{B_{\mathrm{ECR}}}=$ 0.60 , typical instability threshold with $\frac{B_{\min }}{B_{\mathrm{ECR}}}=0.73$, and unstable operation regime with $\frac{B_{\min }}{B_{\mathrm{ECR}}}=0.80$. The $B_{\min } / B_{\mathrm{ECR}}-$ ratios are given for cold electrons. The figure shows that

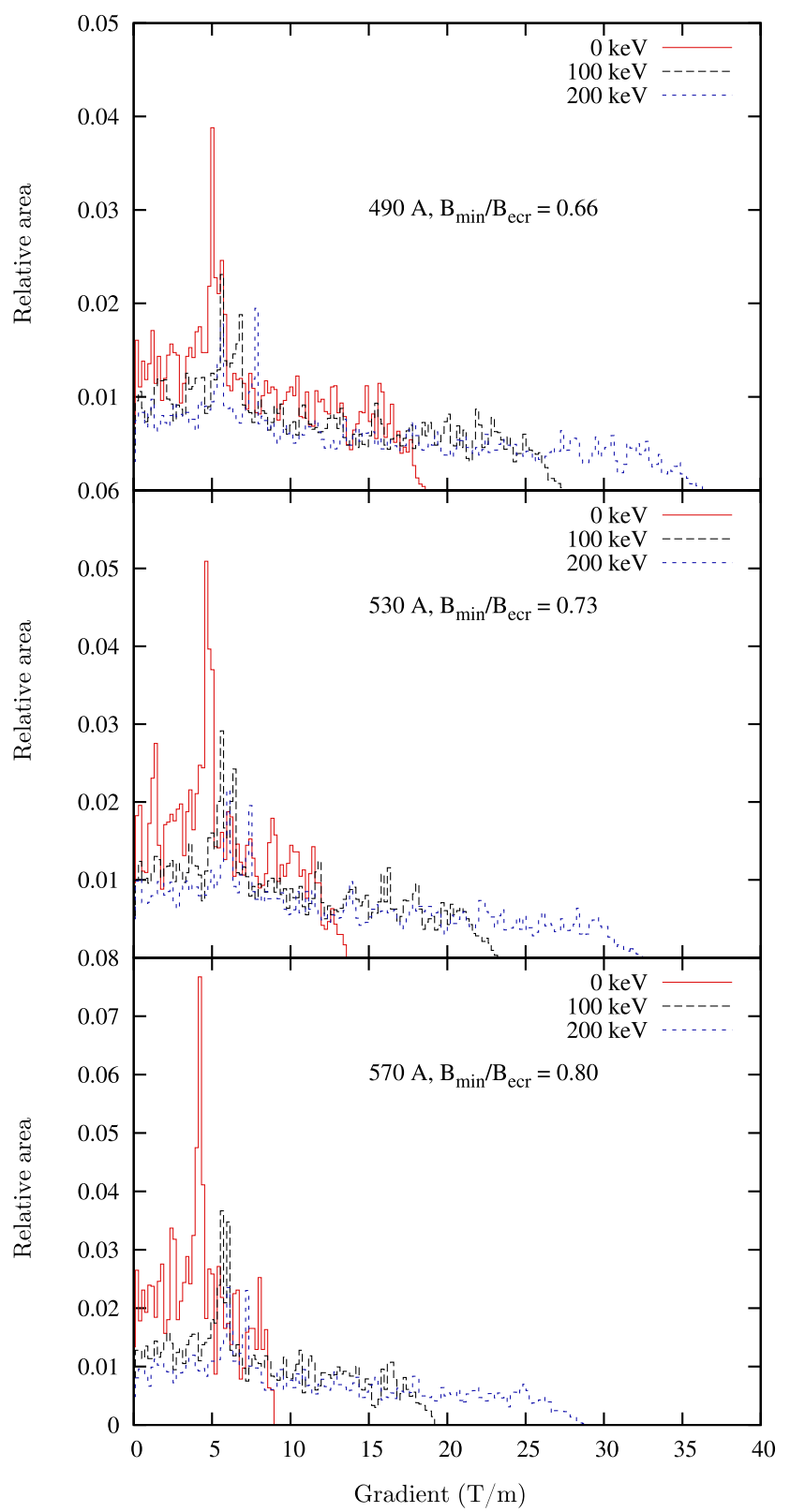

FIG. 7. Distribution of the parallel component of the magnetic field gradient at the resonance for different magnetic field configurations and electron energies. Each histogram has been normalized by the area. Further details of the magnetic field model used for creating the plots can be found from the Appendix. 

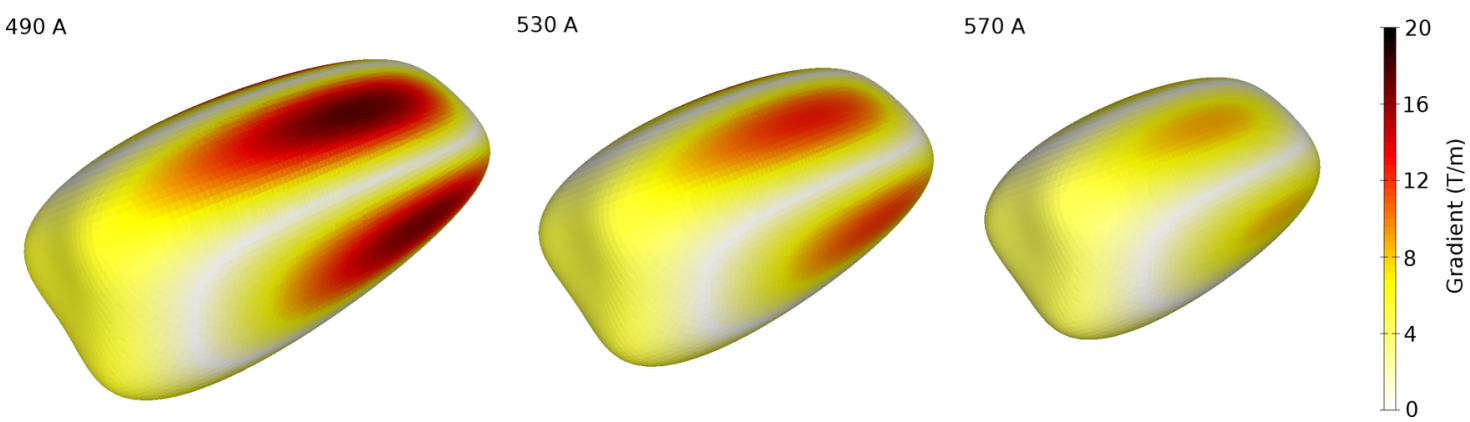

FIG. 8. The spatial distribution of the parallel magnetic field gradient on the cold electron ECR-surface with different coil currents of the JYFL 14 GHz ECRIS. The surfaces are visualized with constant projection which makes the changes of the resonance volume observable.

the distribution of the parallel resonance gradient component shifts drastically toward lower values with increasing solenoid current. The effect is pronounced for cold electrons.

The variation of the parallel magnetic field gradient component with the coil currents is further illustrated in Fig. 8 showing a color map of the gradient on the ECRsurface for cold electrons with three different coil currents corresponding to stable operation regime (490 A), typical instability threshold field (530 A), and unstable operation regime $(570 \mathrm{~A})$. It can be observed that the most drastic changes of the gradient occur near the poles of the sextupole field. This is due to the reduced volume of the ECR-zone with increasing coil current and the $r^{2}$-dependence of the sextupole field strength, which causes the maximum value of the parallel gradient component to decrease by a factor $>2$ within the presented range of solenoid coil currents.

Finally, Table I provides a summary of the effect of solenoid coil currents on the magnetic field of the JYFL $14 \mathrm{GHz}$ ECRIS. The data are displayed in order to link the described variation of the coil currents with characteristic figures of the magnetic field topology. It is of note that the average (parallel) gradient on the resonance surface changes almost $50 \%$ while the on-axis gradients change only by approximately $20 \%$ in the given range of the coil currents.

The results presented in this paper imply that electron cyclotron instabilities limit the JYFL $14 \mathrm{GHz}$ ECRIS performances in terms of high charge state ion beam production and

TABLE I. JYFL 14 GHz ECRIS solenoid coil current (injection and extraction) [A], corresponding magnetic field strengths (injection, extraction) [T], $\frac{B_{\min }}{B_{\mathrm{ECR}}}$, on-axis gradients at the resonance $[\mathrm{T} / \mathrm{m}]$, parallel gradient averaged over the ECR surface $[\mathrm{T} / \mathrm{m}]$, distance between the resonance points on axis $[\mathrm{mm}]$, area of the ECR surface $\left[\mathrm{cm}^{2}\right]$, and the volume enclosed by the ECR surface $\left[\mathrm{cm}^{3}\right]$. Data calculated for cold electrons.

\begin{tabular}{lccc}
\hline \hline & Stable regime & Instability threshold & Unstable regime \\
\hline Coil currents & $490 / 490$ & $530 / 530$ & $570 / 570$ \\
$B_{\text {inj }} / B_{\text {ext }}$ & $1.98 / 0.90$ & $2.06 / 0.96$ & $2.13 / 1.02$ \\
$B_{\min }$ & 0.66 & 0.73 & 0.80 \\
$B_{\mathrm{ECR}}$ & $7.6 / 6.7$ & $6.9 / 6.3$ & $5.9 / 5.6$ \\
$\nabla B_{\text {inj }} / \nabla B_{\text {ext }}$ & 7.5 & 5.6 & 4.0 \\
$\left\langle\nabla B_{\mathrm{ECR}}\right\rangle_{\|}$ & 91 & 79 & 67 \\
$L_{\mathrm{ECR}}$ & 176 & 140 & 110 \\
$A_{\mathrm{ECR}}$ & 181 & 134 & 97 \\
$V_{\mathrm{ECR}}$ & & & \\
\hline \hline
\end{tabular}

optimization. It has been found that the onset of the instabilities typically occurs at $B_{\min } / B_{\mathrm{ECR}}$-ratio of $0.7-0.75$ while at $\frac{B_{\min }}{B_{\mathrm{ECR}}}=$ 0.80 , referred as the optimum value by the ECRIS scaling laws, ${ }^{5}$ the plasma always exhibits periodic instabilities at several $\mathrm{kHz}$ repetition rate under "normal" operation conditions, i.e., at pressure $<2 \cdot 10^{-6} \mathrm{mbar} /$ power $>50 \mathrm{~W}$. According to our knowledge, no other explanation for the observed upper limit of B-minimum has been demonstrated experimentally. It has been suggested by Girard et al. ${ }^{33}$ that due to the reduced ionization rate by the hot electrons the source performance deteriorates if the electron heating is too efficient. Gammino et al. ${ }^{8}$ have used this explanation for the decreased source performance at $\frac{B_{\min }}{B_{\mathrm{ECR}}} \geq 0.75$. The ionization rate, however, is a smooth function of the average electron energy. The decrease of the extracted beam currents of highly charged ions discussed here exhibits threshold behavior typical to non-linear effects, i.e., instabilities. Moreover, the increase of low charge state ion currents at high $B_{\min } / B_{\mathrm{ECR}}$-ratios cannot be explained by increasing average electron energy with decreasing magnetic field gradient. Thus, it is concluded that kinetic instabilities are the most probable reason for the observed behavior. Nakagawa et $a l .{ }^{27}$ have used laser ablation technique to study the variations of the plasma density and energy of the warm electron population as a function of the $B_{\min } / B_{\mathrm{ECR}}$-ratio in a $18 \mathrm{GHz}$ ECRIS. It was observed that the density of the warm electrons tends to decrease when $\frac{B_{\min }}{B_{\mathrm{ECR}}} \geq 0.75$ accompanied by an increase of neutral gas pressure. These effects were explained by reduced power absorption due to smaller resonance area and subsequent variation of so-called ion pumping affecting the neutral particle density in the plasma core. It has been observed with the JYFL $14 \mathrm{GHz}$ ECRIS through optical emission spectroscopy ${ }^{14}$ that the neutral gas density in the plasma increases as a consequence of the kinetic instabilities discussed here. Furthermore, the decreased electron density can be explained by instability-induced electron losses and enhanced damping of electron energy in inelastic collisions. It is, therefore, argued that the data presented by Nakagawa $\mathrm{et} \mathrm{al.}{ }^{27}$ are in good agreement with the results obtained with the JYFL 14 GHz ECRIS. Several researchers have reported that double frequency operation enhances ECRIS performances through improved plasma stability. ${ }^{34-36}$ In double frequency heating mode, the optimum $B_{\min } / B_{\mathrm{ECR}}$-ratio for the secondary (lower) frequency is often $\geq 0.9$. However, the exact mechanism responsible for mitigating the effects of plasma instabilities and, thus, 
extending the parameter space available for high charge state ion current optimization in double frequency heating mode is unknown.

The obtained data can be used for guiding the design of future ECR ion sources as it seems that the average parallel component of the magnetic field gradient on the ECR-surface for cold electrons should exceed $6 \mathrm{~T} / \mathrm{m}$ for stable operation at $14 \mathrm{GHz}$ primary frequency. It is worth noting that the instability growth rate depends directly on the B-field, ${ }^{15,37}$ which implies that also the absolute magnitude of the field could contribute to the onset of the instabilities together with the field gradient. Complementary experiments with ECR ion sources operating at higher frequencies should be conducted to confirm these conclusions. Such experiments can be carried out best with superconducting ECRISs enabling independent adjustment of solenoid and sextupole fields as well as operation in multiple frequency heating mode.

\section{ACKNOWLEDGMENTS}

This work has been supported by the EU 7th framework programme "Integrating Activities-Transnational Access," Project No. 262010 (ENSAR), Magnus Ehrnrooth foundation, the Academy of Finland under the Finnish Centre of Excellence Programme 2012-2017 (Nuclear and Accelerator Based Physics Research at JYFL) and researcher mobility Grant Nos. 267174 and 267227, the Russian Foundation for Basic Research Grant Nos. 12-02-31206 and 13-02-00951, the Russian Academy of Sciences program OFN-15 and Presidential RSS Council Grant (No. SP-23.2012.1). Work of V. Skalyga was supported by the grant of the President of Russian Federation for young scientists (MK-6565.2014.2).

\section{APPENDIX: MAGNETIC FIELD MODEL}

The model used for characterizing the magnetic field, e.g., the distribution of field gradient is a superposition of analytical fits of the solenoid field in $(r, z)$ coordinates and the sextupole field in $(r, \theta)$ coordinates. The solenoid field model is constructed by fitting a sixth order polynomial $B_{z}(z)$ to onaxis magnetic field data computed with FEMM 4.2. ${ }^{38}$ The off-axis solenoid field is evaluated with a standard expansion, presented, for example, in Ref. 39, i.e.,

$$
\begin{aligned}
B_{z}(r, z)= & A_{0}+A_{1} z+A_{2}\left(z^{2}-\frac{r^{2}}{2}\right)+A_{3}\left(z^{3}-\frac{3 r^{2} z}{2}\right) \\
& +A_{4}\left(z^{4}-3 r^{2} z^{2}+\frac{3 r^{4}}{8}\right)+A_{5}\left(z^{5}-5 r^{2} z^{3}+\frac{15 r^{4} z}{8}\right) \\
& +A_{6}\left(z^{6}-\frac{15 r^{2} z^{4}}{2}+\frac{45 r^{4} z^{2}}{8}-\frac{5 r^{6}}{16}\right), \\
B_{r}(r, z)= & -A_{1} \frac{r}{2}-A_{2} r z-A_{3}\left(\frac{3 r z^{2}}{2}-\frac{3 r^{3}}{8}\right) \\
& -A_{4}\left(2 r z^{3}-\frac{3 r^{3} z}{2}\right)-A_{5}\left(\frac{5 r z^{4}}{2}-\frac{15 r^{3} z^{2}}{4}+\frac{5 r^{5}}{16}\right) \\
& -A_{6}\left(3 r z^{5}-\frac{15 r^{3} z^{3}}{2}+\frac{15 r^{5} z}{8}\right),
\end{aligned}
$$

where the values $A_{0}$ through $A_{6}$ are determined from the leastsquare fits of the simulation data with $r=0$ at various solenoid coil currents.

The sextupole field model is constructed by fitting a linear combination of cylindrical multipoles,

$$
\begin{aligned}
& B_{r}(r, \theta)=\sum_{i} J_{i}\left(\frac{r}{r_{\text {ref }}}\right)^{i-1} \cos (i \theta), \\
& B_{\theta}(r, \theta)=\sum_{i}-J_{i}\left(\frac{r}{r_{\text {ref }}}\right)^{i-1} \sin (i \theta),
\end{aligned}
$$

to field data simulated with FEMM 4.2. A six-parameter fit is acquired by taking into account the first non-zero parameters $J_{3}, J_{9}, J_{15}, J_{21}, J_{27}$, and $J_{33}$. The parameters $J_{1}, J_{2}, J_{4}, \ldots$ are zero due to sextupole symmetry. The reference radius $r_{\text {ref }}$ corresponds to the plasma chamber radius of $38 \mathrm{~mm}$.

Using the analytical superposition of the solenoid and sextupole fields allows computing the magnetic flux density and magnetic field gradient distributions corresponding to various coil currents in reasonable time as opposed to time-consuming 3D simulations with limited data export capabilities. The error of the resulting data fits in comparison to simulated data is $\leq 3 \%$ within the region of interest in the plasma chamber volume.

${ }^{1} \mathrm{R}$. Geller, Electron Cyclotron Resonance Ion Sources and ECR Plasmas (IOP Publishing Ltd, 1996).

${ }^{2}$ R. Geller, T. Lamy, and P. Sortais, Rev. Sci. Instrum. 77, 03 B107 (2006).

${ }^{3}$ T. Antaya and S. Gammino, Rev. Sci. Instrum. 65, 1060 (1994).

${ }^{4}$ The Physics and Technology of Ion Sources, edited by I. G. Brown (John Wiley \& Sons, Inc., 2004).

${ }^{5}$ D. Hitz, A. Girard, G. Melin, S. Gammino, G. Ciavola, and L. Celona, Rev. Sci. Instrum. 73, 509 (2002).

${ }^{6}$ M. C. Williamson, A. J. Lichtenberg, and M. A. Lieberman, J. Appl. Phys. 72(9), 3924 (1992).

${ }^{7}$ H. Koivisto, Rev. Sci. Instrum. 70, 2979 (1999).

${ }^{8}$ S. Gammino, D. Mascali, L. Celona, F. Maimone, and G. Ciavola, Plasma Sources Sci. Technol. 18, 045016 (2009).

${ }^{9}$ C. Barue, M. Lamoreux, P. Briand, A. Girard, and G. Melin, J. Appl. Phys. 76, 5 (1994).

${ }^{10}$ G. Douysset, H. Khodja, A. Girard, and J. P. Briand, Phys. Rev. E 61(3), 3015 (2000).

${ }^{11}$ D. Leitner, J. Y. Benitez, C. M. Lyneis, D. S. Todd, T. Ropponen, J. Ropponen, H. Koivisto, and S. Gammino, Rev. Sci. Instrum. 79, 033302 (2008).

${ }^{12}$ S. V. Golubev and A. G. Shalashov, Phys. Rev. Lett. 99, 205002 (2007).

${ }^{13} \mathrm{G}$. Melin et al., Proceedings of the 10th International Workshop on ECR Ion Sources, CONF-9011136 (ORNL, Knoxville, TN, 1991), p. 1.

${ }^{14}$ O. Tarvainen, I. Izotov, D. Mansfeld, V. Skalyga, S. Golubev, T. Kalvas, H. Koivisto, J. Komppula, R. Kronholm, J. Laulainen, and V. Toivanen, Plasma Sources Sci. Technol. 23, 025020 (2014).

${ }^{15}$ S. V. Golubev and A. G. Shalashov, J. Exp. Theor. Phys. Lett. 86, 2 (2007).

${ }^{16} \mathrm{~V}$. Yu. Trakhtengerts and M. J. Rycroft, Whistler and Alfvén Mode Cyclotron Masers in Space (Cambridge University Press, 2008), p. 36.

${ }^{17}$ V. L. Ginzburg, The Propagation of Electromagnetic Waves in Plasmas (Pergamon press, 1970).

${ }^{18}$ V. Toivanen, O. Tarvainen, J. Komppula, and H. Koivisto, J. Instrum. 8, T02005 (2013).

${ }^{19}$ H. Koivisto, P. Heikkinen, V. Hänninen, A. Lassila, H. Leinonen, V. Nieminen, J. Pakarinen, K. Ranttila, J. Ärje, and E. Liukkonen, Nucl. Instrum. Methods B 174, 379 (2001).

${ }^{20}$ O. Tarvainen, T. Kalvas, H. Koivisto, T. Ropponen, V. Toivanen, J. H. Vainionpää, A. Virtanen, and J. Ärje, in Proceedings of HIAT 2009, Venice, Italy, 8-12 June 2010, http://JACoW.org.

${ }^{21}$ T. Ropponen, O. Tarvainen, P. Jones, P. Peura, T. Kalvas, P. Suominen, H. Koivisto, and J. Ärje, Nucl. Instrum. Methods Phys. Res., Sect. A 600, 525 (2009).

${ }^{22}$ T. Ropponen, O. Tarvainen, P. Jones, P. Kalvas, T. Suominen, and P. Koivisto, IEEE Trans. Plasma Sci. 37, 2146 (2009) 
${ }^{23}$ O. Tarvainen, T. Kalvas, H. Koivisto, J. Komppula, R. Kronholm, J. Laulainen, I. Izotov, D. Mansfeld, and V. Skalyga, Proceedings on the 21st International Workshop on ECR Ion Sources, ECRIS14, Nizhny-Novgorod, Russia, 2014 (to be published), http://JACoW.org.

${ }^{24}$ V. Toivanen, T. Kalvas, H. Koivisto, J. Komppula, and O. Tarvainen, J. Instrum. 8, P05003 (2013).

${ }^{25}$ H. Arai, M. Imanaka, S. M. Lee, Y. Higurashi, T. Nakagawa, M. Kidera, T. Kageyama, M. Kase, Y. Yano, and T. Aihara, Nucl. Instrum. Methods Phys. Res., Sect. A 491, 1-2 (2002).

${ }^{26}$ D. Leitner, C. M. Lyneis, T. Loew, D. S. Todd, S. Virostek, and O. Tarvainen, Rev. Sci. Instrum. 77(3), 03A342 (2006).

${ }^{27}$ T. Nakagawa, M. Kidera, Y. Higurasi, J. Ohnishi, T. Kageyama, T. Aihara, A. Goto, and Y. Yano, High Energy Phys. Nucl. Phys. 31(1), (2007).

${ }^{28}$ T. Rognlien and T. Cutler, Nucl. Fusion 20 (1980), p. 1003.

${ }^{29}$ O. Tarvainen, T. Kalvas, H. A. Koivisto, J. Komppula, V. Toivanen, C. M. Lyneis, and M. M. Strohmeier, in TUXO02, Proceedings on the 20th International Workshop on ECRIS, Sydney, Australia, 25-28 September 2012, http://JACoW.org, p. 5.
${ }^{30}$ R. C. Vondrasek, R. H. Scott, R. C. Pardo, and D. Edgell, Rev. Sci. Instrum. 73, 548 (2002).

${ }^{31}$ C. Perret, A. Girard, H. Khodja, and G. Melin, Phys. Plasmas 73, 3408 (1999).

${ }^{32}$ A. Girard, C. Pernot, and G. Melin, Phys. Rev. E 62, 1 (2000).

${ }^{33}$ A. Girard, C. Perret, G. Melin, and C. Lecot, Rev. Sci. Instrum. 69, 1100 (1998).

${ }^{34}$ Z. Q. Xie and C. M. Lyneis, 12th International Workshop on ECR Ion Sources, Riken, Japan, 25-27 April, 1995.

${ }^{35}$ R. C. Vondrasek, R. Scott, and R. C. Pardo, Rev. Sci. Instrum. 75, 1532 (2004).

${ }^{36}$ A. Kitagawa, T. Fujita, M. Muramatsu, S. Biri, R. Racz, Y. Kato, K. Yano, N. Sasaki, and W. Takasugi, in TUXO03, Proceedings on the 20th International Workshop on ECRIS, Sydney, Australia, 25-28 September 2012, http://JACoW.org, p. 10.

${ }^{37}$ A. G. Demekhov, Radiophys. Quantum Electron. 30, 547 (1987).

${ }^{38}$ D. C. Meeker, Finite Element Method Magnetics, Version 4.2, http://www. femm.info.

${ }^{39}$ J. Rodney and M. Vaughan, IEEE Trans. Electron Devices 19, 144 (1972). 